\title{
Fungos endofíticos associados a plantas medicinais
}

\author{
MUSSI-DIAS, V. ${ }^{1 *}$; ARAÚJO, A.C.O. ${ }^{1}$; SILVEIRA, S.F. ; ROCABADO, J.M.A. ${ }^{2}$; ARAÚJO, K.L. ${ }^{3}$ \\ ${ }^{1}$ Laboratório de Entomologia e Fitopatologia/CCTA - Universidade Estadual do Norte Fluminense Darcy Ribeiro, \\ UENF, CEP: 28013-602, Campos dos Goytacazes-Brasil ' ${ }^{2}$ Universidade Federal do Reconcavo da Bahia, CCAAB, \\ CEP: 44380-000, Cruz das Almas-Brasil ${ }^{3}$ Universidade do Estado de Mato Grosso, UNEMAT, Departamento de \\ Agronomia, Laboratório de Resistência Genética, CEP: 78200-000, Cáceres-Brasil *vicmussi@uenf.br
}

\begin{abstract}
RESUMO: Com a utilização de plantas medicinais em infusões, xaropes, tinturas, ungüentos, dentre outras formas, pressupõe-se que fungos endofíticos, presentes no interior das plantas, mas sem causar doença, possam tornar-se um componente destes produtos, principalmente quando utilizados in natura. Além disso, os fungos endofíticos podem também produzir substâncias tóxicas aos usuários ou mesmo alterar o metabolismo vegetal, modificando a composição e as propriedades medicinais, assim como, a qualidade do produto armazenado e comercializado. Neste sentido, objetivou-se isolar e identificar a flora fúngica endofítica de onze espécies medicinais escolhidas ao acaso. Obtiveram-se culturas-puras dos fungos Phomopsis, Colletotrichum, Pestalotia, Trichoderma, Fusarium, Nigrospora e Glomerella ocorrendo endofiticamente em Plectranthus barbatus, Vernonia condensata, Pfaffia paniculata, Foeniculum vulgare, Cymbopogon citratus, Cymbopogon nardus, Cordia curassavica, Maytenus ilicifolia, Punica granatum, Morus nigra e Bauhinia forficata. As espécies vegetais em que se identificaram o maior número de fungos endofíticos foram Vernonia condensata, Punica granatum e Morus nigra. Todos os fungos recuperados neste trabalho apresentaram características estritamente endofíticas, não manifestando patogenicidade nas espécies hospedeiras. Dentre os fungos detectados, especial atenção deve ser dada ao gênero Fusarium, uma vez que inúmeras espécies deste gênero são conhecidas produtoras de micotoxinas e constituem-se em importantes patógenos pós-colheita.
\end{abstract}

Palavras-chave: fungos endofíticos, plantas medicinais

ABSTRACT: Endophytic fungi associated with medicinal plants. With the use of medicinal plants in infusions, syrups, dyes, unguents, among other forms, it is expected that endophytic fungi, present inside the plants but not causing diseases, become components of these products, especially when used in natura. In addition, endophytic fungi can produce toxic substances to the users or even modify the plant metabolism, altering the medicinal composition and properties, as well as the quality of the stored and commercialized product. Therefore, the aim of this study was to isolate and identify the endophytic flora from eleven randomly chosen medicinal species. Pure cultures were obtained from the fungi Phomopsis, Colletotrichum, Pestalotia, Trichoderma, Fusarium, Nigrospora and Glomerella endophytically occurring in Plectranthus barbatus, Vernonia condensata, Pfaffia paniculata, Foeniculum vulgare, Cymbopogon citratus, Cymbopogon nardus, Cordia curassavica, Maytenus ilicifolia, Punica granatum, Morus nigra and Bauhinia forficata. The plant species that presented the highest number of endophytic fungi were Vernonia condensata, Punica granatum and Morus nigra. All fungi recovered in this study showed strictly endophytic features, not manifesting pathogenicity in their host species. Among the detected fungi, special attention must be given to the genus Fusarium, since a wide range of species of this genus are known to produce mycotoxins and constitute important post-harvest pathogens.

Key words: Endophytic fungi, medicinal plants

Recebido para publicação em 16/09/2009 Aceito para publicação em 16/03/2012

Rev. Bras. PI. Med., Botucatu, v.14, n.2, p.261-266, 2012. 


\section{INTRODUÇÃO}

As plantas medicinais no Brasil podem ser utilizadas de diversas maneiras e com diferentes propósitos, seja in natura, com partes inteiras ou sob a forma para preparação de chás e/ou outros preparos caseiros. São destinadas ao uso doméstico ou em rituais religiosos, espirituais de cura e transcendência sob a forma pulverizada, extratos brutos ou frações enriquecidas, extratos padronizados, tinturas, extratos fluidos, pós, comprimidos, cápsulas, e, finalmente, podem ser submetidas a sucessivos processos de extração e purificação, para isolamento das substâncias de interesse (Rates, 2001).No entanto, o uso de plantas medicinais de forma indiscriminada ou negligenciada pode ser potencialmente agressivo, devendo-se ter cautela e respeitar os riscos de toxidez. Um exemplo disso é o efeito hepatotóxico de apiol, safrol, lignanas e alcalóides bem como a ação tóxica renal causada por espécies vegetais que contém terpenos e saponinas e alguns tipos de dermatites, causadas por espécies ricas em lactonas sesquiterpênicas e produtos naturais do tipo furanocumarinas (Veiga Júnior et al., 2005).

Os microrganismos endofíticos, tais como os fungos, podem colonizar folhas, ramos e raízes, sem causar prejuízos aos hospedeiros (Peixoto Neto et al., 2002), habitando de forma sistêmica o apoplasto, vasos condutores e em alguns casos o interior da célula (Azevedo et al., 2003). Dessa relação simbiótica os fungos podem desempenhar funções relevantes para sanidade vegetal, protegendo as plantas contra pragas e patógenos, aumentando $o$ crescimento, enraizamento, resistência a estresses, além de produzir compostos químicos como enzimas, alcalóides, hormônios e antibióticos (Peixoto Neto et al., 2002). Estes compostos, por sua vez, podem apresentar considerável toxicidade, como é o caso de alcalóides produzidos por fungos endofíticos os quais são responsáveis pela proteção das plantas, especialmente gramíneas forrageiras, contra animais herbívoros (Azevedo, 1999). Entretanto, o efeito benéfico da associação planta-endófito, é muito maior que os prejudiciais e é devido a isso que estes microrganismos vêm se tornando importante ferramenta para a agricultura moderna (Peixoto Neto et al., 2002). O fungo endofítico Taxomyces andreanae Strobel, A. Stierle, D. Stierle \& W.M. Hess isolado a partir de Taxus brevifolia Nutt. (Taxaceae), é capaz de produzir o complexo diterpenóide Taxol, utilizado no combate de vários tipos de câncer (Stierle et al., 1993). Outros fungos isolados de plantas do gênero Taxus L. também têm a capacidade de produzir taxol (Peixoto Neto et al., 2004). O fungo Stegolerium kukenani Strobel, W.M. Hess \& E.J. Ford endofítico da espécie tropical Stegolepis guianensis Klotzsch ex Körn., uma Rapateaceae nativa de Roraima (Brasil) e Venezuela, é outro exemplo de fungo endofítico capaz de produzir o complexo diterpenóide Taxol (Strobel et al., 2001). Do ponto de vista ecológico, tal descoberta é extremamente importante, pois pode minimizar o perigo de extinção de algumas espécies vegetais as quais são coletadas para a extração de produtos medicinais, garantindo assim a preservação destas e, mantendo a produção de compostos que garantam a continuidade no tratamento de pessoas afetadas por inúmeras doenças (Souza, 2001; Peixoto Neto et al., 2002; Peixoto Neto et al., 2004).

Devido as poucas informações na literatura especializada sobre a presença, interferência ou alterações das características, principalmente in natura das plantas medicinais comumente utilizadas, objetivou-se neste trabalho isolar e identificar os fungos endofíticos de algumas espécies medicinais a fim de se conhecer a abundância e a diversidade desses organismos no material estudado.

\section{MATERIALE MÉTODO}

Coletaram-se folhas frescas de coleção de espécies medicinais mantidas na Unidade de Apoio à Pesquisa do Centro de Ciências e Tecnologias Agropecuárias localizada no "Campus Leonel Brizola" da Universidade Estadual do Norte Fluminense Darcy Ribeiro - Campos dos Goytacazes/RJ, em 2006. Amostras de onze espécies foram colhidas, optandose por aquelas sem sintomas de doenças (Tabela 1).

Folhas assintomáticas totalmente expandidas foram destacadas, acondicionadas em sacolas de papel e levadas para o laboratório. Cada folha foi lavada com detergente neutro, sob água corrente para a retirada do excesso de impurezas e da flora epifítica residente (Souza et al., 2004). Posteriormente, o material foi colocado sobre papel toalha para a retirada do excesso de água.

Com auxílio de furador de rolha $(1,3 \mathrm{~cm}$ de diâmetro) retiraram-se fragmentos do limbo foliar. Esses fragmentos foram imersos em álcool 70\% (1 min) para quebrar a tensão superficial, hipoclorito de sódio 3\% (3 min) para desinfestação, álcool 70\% (30 seg) para retirar o excesso de hipoclorito e, água destilada estéril para lavagem final do fragmento (Souza et al., 2004). O excesso de água de cada fragmento foi absorvido por papel de filtro previamente esterilizado. Todo o procedimento foi realizado sob câmara asséptica.

De cada espécie vegetal seis fragmentos da amostra assim preparados foram semeados em duas placas de petri contendo meio de cultura semi-sólido "BDA" (batata, dextrose e ágar), acrescido de 0,2\% de extrato de levedura e solução estoque de sulfato de estreptomicina (100 $\mathrm{mL} \mathrm{L}^{-1}$ ) (modificado de Freire \& Bezerra, 2001 e de Souza et. al., 2004). Após a vedação das placas com filme transparente de "PVC",

Rev. Bras. PI. Med., Botucatu, v.14, n.2, p.261-266, 2012. 
TABELA 1. Espécies vegetais utilizadas na obtenção de fungos endofíticos e respectivas famílias.

\begin{tabular}{llll}
\hline Nome científico & Nome popular & Família botânica & Local de coleta* \\
\hline Plectranthus barbatus Andrews & Boldo & Lamiaceae & UAP/UENF \\
Vernonia condensata Baker & Boldo-do-chile & Asteraceae & UAP/UENF \\
Pfaffia paniculata (Mart.) Kuntze & Fáfia & Amaranthaceae & UAP/UENF \\
Foeniculum vulgare Mill. & Funcho & Apiaceae & UAP/UENF \\
Cymbopogon citratus (DC.) Stapf. & Capim-limão & Poaceae & UAP/UENF \\
Cymbopogon nardus (L.) Rendle & Citronela & Poaceae & UAP/UENF \\
Cordia curassavica (Jacq.) Roem. \& Schult. & Erva-baleeira & Boraginaceae & UAP/UENF \\
Maytenus ilicifolia Mart. ex Reissek & Espinheira-santa & Celastraceae & UAP/UENF \\
Punica granatum L. & Romã & Lythraceae & UAP/UENF \\
Morus nigra L. & Amora-negra & Moraceae & UAP/UENF \\
Bauhinia forficata Link. & Pata-de-vaca & Fabaceae & UAP/UENF \\
\hline
\end{tabular}

* Unidade de apoio à pesquisa do Centro de Ciências e Tecnologias Agropecuárias, localizada no Campus Leonel Brizola da Universidade Estadual do Norte Fluminense Darcy Ribeiro/UENF - Campos dos Goytacazes, RJ.

as mesmas foram colocadas em câmaras de crescimento do tipo "BOD" a $28^{\circ} \mathrm{C}$, com fotoperíodo de 12 horas.

Sete dias após a incubação foi iniciada a identificação das colônias fúngicas crescidas e esporuladas. Para tanto, foram confeccionadas lâminas descartáveis dos fungos utilizando-se lactofenol com ou sem corante azul de algodão (Dhingra \& Sinclair, 1994).

As observações foram feitas em microscópio óptico de luz e os fungos foram identificados com base na morfologia conjunta das estruturas reprodutivas de acordo com literatura especializada (Sutton, 1980; Hanlin \& Menezes, 1996; Barnett \& Hunter, 1998).

\section{RESULTADO E DISCUSSÃO}

Foram obtidos 20 isolados fúngicos sendo seis do gênero Phomopsis Sacc. \& Roum., cinco Colletotrichum Corda, cinco Nigrospora Zimm., um de Glomerella Spauld. \& H. Schrenk, um de Fusarium Link, um de Pestalotia De Not. e um de Trichoderma Pers.. De modo geral houve maior frequência de fungos endofíticos nas folhas de amora-negra, boldodo-chile e romã, seguidos de erva-baleeira e demais espécies de plantas (Tabela 2). Não há registros na literatura sobre a ocorrência de endofíticos nestas espécies embora os fungos endofíticos aqui recuperados já tenham sido reportados em inúmeros hospedeiros e diversas localidades do mundo (Stone et al., 2004).

Das folhas das plantas amostradas não foi isolado nenhum fungo endofitico para o capim-limão. Já para as folhas de amora-negra, obteve-se isolados de Phomopsis sp. e outro fungo da classe
Coelomycetes, não identificado (Tabela 2). Estes dados sugerem flutuação espacial e sazonal dos endofiticos nestas espécies vegetais o que também foi observado por Gao et al. (2005) em Heterosmilax japonica. Com essas observações, confirma-se que a mesma espécie de fungo endofítico pode ocorrer ou não em mesma espécie vegetal, dependendo do local de cultivo, condições ambientais, estádio fenológico da planta e nível tecnológico empregado.

Isolamentos efetuados a partir de outras espécies hospedeiras também podem resultar nos mesmos endófitos aqui encontrados. Como exemplos podem ser citados Phomopsis e Glomerella em Heterosmilax japonica Kunt (Gao et al., 2005), Colletotrichum, Glomerella e Trichoderma em Palicourea longiflora DC. e Strychnos cogens Benth. (Souza et al., 2004); Fusarium dentre outras em Bactris gasipaes Kunt (pupunheira) (Almeida et al., 2005). Alta frequência de Colletotrichum gloeosporioides (Penz.) Penz. \& Sacc. foi encontrada em Copernicia prunifera (Mill.) H.E. Moore, Anacardium occidentale L., Mangifera indica L., Spondias purpurea L., Spondias tuberosa Arruda, Manihot esculenta Crantz, Malpighia emarginata DC., Punica granatum L. e Ricinus communis L. (Freire \& Bezerra, 2001). Os mesmos autores encontraram baixa freqüência de Phomopsis sp. em Copernicia prunifera, Mangifera indica, Spondias purpurea, Spondias tuberosa, Punica granatum e Syzygium jambolanum (Lam.) DC. assim como de Nigrospora sp. em Spondias purpurea, Spondias tuberosa e Malpighia emarginata bem como de Fusarium em Copernicia prunifera e Anacardium occidentale. Kamei et al. (2008) isolaram Phomopsis sp. em Borreria verticillata (L.) G. Mey. (vassourinha-de-botão) e Pimentel et al. (2006), Colletotrichum, Fusarium e 
TABELA 2. Gêneros de fungos endofíticos isolados das respectivas espécies hospedeiras.

\begin{tabular}{|c|c|c|c|}
\hline Espécie vegetal & Nome comum & Isolado & Fungos endofíticos recuperados \\
\hline Plectranthus barbatus & Boldo & UENF/CF 60 & Phomopsis sp. \\
\hline \multirow[t]{3}{*}{ Vernonia condensata } & Boldo-do-chile & UENF/CF 64 & Colletotrichum sp. \\
\hline & & UENF/CF 61 & Phomopsis sp. \\
\hline & & UENF/CF 63 & Pestalotia sp. \\
\hline Pfaffia paniculata & Fáfia & UENF/CF 65 & Phomopsis sp. \\
\hline Cymbopogon nardus & Citronela & UENF/CF 80 & Colletotrichum sp. \\
\hline Foeniculum vulgare & Funcho, erva-doce & UENF/CF 81 & Trichoderma sp. \\
\hline Cymbopogon citratus & Capim-limão & UENF/CF 66 & Fusarium sp. \\
\hline \multirow[t]{2}{*}{ Cordia curassavica } & Erva-baleeira & UENF/CF 70 & Phomopsis sp. \\
\hline & & UENF/CF 69 & Nigrospora sp. \\
\hline Maytenus ilicifolia & Espinheira-santa & UENF/CF 71 & Nigrospora sp. \\
\hline \multirow[t]{3}{*}{ Punica granatum } & Romã & - & Colletotrichum sp \\
\hline & & - & Phomopsis sp. \\
\hline & & - & Nigrospora sp. \\
\hline \multirow[t]{4}{*}{ Morus nigra } & Amora-negra & UENF/CF $75 a$ & Glomerella sp. \\
\hline & & UENF/CF 75b & Colletotrichum sp \\
\hline & & UENF/CF 73 & Phomopsis sp. \\
\hline & & UENF/CF 74 & Nigrospora sp. \\
\hline \multirow[t]{2}{*}{ Bauhinia forficata } & Pata-de-vaca & UENF/CF 77 & Colletotrichum sp. \\
\hline & & UENF/CF 78 & Nigrospora sp. \\
\hline
\end{tabular}

Trichoderma em Ilex paraguariensis A. St.-Hil. (ervamate). Ainda Siqueira (2008) encontrou Colletotrichum gloeosporioides, Phomopsis e Fusarium em Lippia sidoides Cham.

Espécies de fungos dos gêneros Colletotrichum, Phomopsis, Nigrospora e Fusarium são agentes etiológicos de doenças em várias espécies de plantas (Kimati et al., 2005). Espécies do gênero Colletotrichum sp. são citadas como agentes etiológicos de inúmeras doenças em hortaliças, fruteiras em geral, podridões de floração e póscolheita. Phomopsis sp. tem sido relatado como agente causal de manchas foliares, queimas, gomose e podridões de frutos. Nigrospora sp. pode causar doenças secundárias em gramíneas e Fusarium sp. ampla gama de doenças em inúmeros hospedeiros, desde murchas vasculares e podridões de colo e raízes até lesões foliares, de floração e frutificação (Kimati et al., 2005). Todos os fungos recuperados neste trabalho se comportaram estritamente como endofíticos não manifestando patogenicidade nas espécies hospedeiras (dados não apresentados de inoculação - teste de patogenicidade).

As interações entre fungos endofíticos e a possível atuação dos mesmos como simbiontes ou mesmo eventuais fitopatógenos nestes hospedeiros necessita mais atenção. Fungos endofíticos podem tornar-se patogênicos em potencial, manifestandose em plantas estressadas e tornando-se problema fitossanitário, como é o caso de Botryosphaeria dothidea (Moug.) Ces. \& De Not. em várias espécies de Eucalyptus L'Hér. no sul da África (Smith et al.,1996), que tornou-se um dos maiores problemas da cultura naquela região. Outro fator que se faz interessante salientar é que devido a desequilíbrios no metabolismo da planta, causados por situações de estresse, sejam elas de causa natural ou mesmo por práticas culturais mal realizadas, como uso excessivo de fertilizantes e outros produtos químicos, pode ocorrer desequilíbrio na micobiota endofítica potencializando-os como fitopatógenos ou favorecendo a ação de fitopatógenos propriamente ditos.

Com relação aos fungos endofíticos Nigrospora, Trichoderma e Pestalotia, encontrados nesse trabalho, não se pode subestimar a capacidade dos mesmos como patógenos em algum momento ou fase de desenvolvimento da cultura, uma vez que ainda não há dados suficientes que comprove a sua estrita relação endofítica.

Vale a pena considerar a presença do gênero Fusarium ocorrendo endofiticamente. Inúmeras espécies deste gênero são potenciais produtoras de micotoxinas (ex.: fumonisina) causadoras de diversos 
distúrbios em animais e humanos (Mallozzi \& Corrêa, 1998), principalmente em material amazenado, podendo levar ao óbto. Mesmo que o processo de desidratação das plantas medicinais colhidas seja feito com o mais rigoroso cuidado e dentro dos padrões de qualidade, a presença de fungos desse tipo, mesmo que invisíveis a olho nú, pode resultar no seu posterior desenvolvimento e produção de toxinas. Portanto, a qualidade do armazenamento, transporte e exposição na prateleira do mercado, principalmente com relação à umidade deve sempre ser observada. Neste sentido, todos os procedimentos que geralmente iniciam-se pela época certa para a colheita, desidratação, acondicionamento, transporte e comercialização (Guião et al., 2004) devem ser seguidos de forma rigorosa para minimizar perdas principalmente de qualidade do produto.

Como a presença de endófitos já foi constatada em plantas medicinais de interesse econômico (Ilex paraguariensis, Borreria G. Mey., Eucalyptus, Heterosmilax japonica), trabalhos verificando a produção de toxinas por estes fungos isoladamente ou em antibiose, comparação de extratos foliares das espécies vegetais com ou sem endófito para avaliar a proteção da planta contra outros endofíticos, insetos, e agentes fitopatogênicos são necessários. Ao ampliar o conhecimento a respeito dessas interações, como agem, os efeitos tóxicos e colaterais e produção de substâncias bioativas pelos mesmos, muitas espécies medicinais que se encontram ameaçadas de extinção, poderão ser preservadas além de possibilitar a síntese de novas substâncias análogas e seletivas.

\section{REFERÊNCIA}

ALMEIDA, C.V.; YARA, R.; ALMEIDA, M. Fungos endofíticos isolados de ápices caulinares de pupunheira cultivada in vivo e in vitro. Pesquisa Agropecuária Brasileira, v.40, n.5, p.467-70, 2005.

AZEVEDO, J.L.; MACCHERONI JÚNIOR, W.; ARAÚJO, W.L. Importância dos microorganismos endofíticos na agricultura. In: LUZ, W.C. et al. (Eds). RAPP: revisão anual de patologia de plantas. v.11. Passo Fundo: Padre Berthier dos Missionários da Sagrada Família, 2003. p.333-71.

AZEVEDO, J.L. Botânica: uma ciência básica ou aplicada?. Revista Biotecnologia Ciência \& Desenvolvimento, v.22, p.225-9, 1999. Disponível em: <http://www.bioteconologia.com.br>. Acesso em: 17 abr. 2006.

BARNETT, H.L.; HUNTER, B.B. Illustrated genera of imperfect fungi. 4.ed. Minnesota: APS Press, 1998. $218 p$.

DHINGRA, O.D.; SINCLAIR, J.B. Basic plant pathology methods. 2.ed. Boca Raton: Lewis Publishers, 1994. 434p.

FREIRE, F.C.O.; BEZERRA, J.L. Foliar endophytic fungi of Ceará State (Brazil): a preliminary study. Summa Phytopathologica, v.27, n.3, p.304-8, 2001.

$\mathrm{GAO}, \mathrm{X} . \mathrm{X}$. et al. High diversity of endophytic fungi from the pharmaceutical plant, Heterosmilax japonica Kunth revealed by cultivation-independent approach. FEMS Microbiology Letters, v.249, p.255-66, 2005.

GUIÃO, M. et al. Plantas medicinais: cultivo, utilidades e comercialização. Belo Horizonte: EMATER/MG/ Prorenda Rural-MG, IEF - MG Doces Matas, 2004. 192p.

HANLIN, R.T.; MENEZES, M. Gêneros ilustrados de ascomicetos. Recife: Imprensa da Universidade Federal Rural de Pernambuco, 1996. 274p.

KAMEI, S.H. et al. Nectria sp., Phomopsis sp. e Xylaria $\mathrm{sp}$. fungos endofíticos isolados de Borreria verticillata (Rubiaceae) L. In: CONGRESSO BRASILEIRO DE GENÉTICA, 54., 2008, Salvador. Resumos ... Salvador, 2008. p.34.

$\mathrm{KIMATI}, \mathrm{H}$. et al. (eds.). Manual de fitopatologia: doenças das plantas cultivadas. 4.ed. São Paulo: Agronômica Ceres, v.2, 2005. 663p.

MALLOZZI, M.A.B.; CORRÊA, B. Fungos toxigênicos e micotoxinas. São Paulo: Instituto Biológico, 1998. 26p. (Boletim Técnico, n.12).

PEIXOTO NETO, P.A.S.; AZEVEDO, J.L.; ARAÚJO, W.L. Microorganismos endofíticos: interação com plantas e potencial biotecnológico. Revista Biotecnologia Ciência \& Desenvolvimento, n.29, p.62-76, 2002. Disponível em: <http://www.biotecnologia. com.br>. Acesso em: 17 abr. 2006.

PEIXOTO NETO, P.A.S.; AZEVEDO, J.L.; CAETANO, L.C. Microrganismos endofíticos em plantas: status atual e perspectivas. Boletín LatinoAmericano y del Caribe de Plantas Medicinales y Aromáticas, v.3, p.67-9, 2004.

PIMENTEL, I.C. et al. Fungos endofíticos em folhas de erva-mate (Ilex paraguariensis A. St.-Hil.). Floresta, v.36, n.1, p.123-8, 2006.

RATES, S.M.K. Promoção do uso racional de fitoterápicos: uma abordagem no ensino da farmacognosia. Revista Brasileira de Farmacognosia, v.11, n.2, p.57-69, 2001. Disponível em: <http://www.farmacognosia.ufpr.br>. Acesso em: 27 jul. 2006.

SIQUEIRA, V.M. Fungos endofíticos de folhas e caule de Lippia sidoides Cham. e avaliação da atividade microbiana. 2008. 94p. Dissertação (Mestrado - Área de concentração em biologia de fungos) - Universidade Federal de Pernambuco, Recife.

SMITH, H.; WINGFIELD, M.J.; PETRINI, O. Botryosphaeria dothidea endophytic in Eucalyptus grandis and Eucalyptus nitens in South Africa. Forest Ecology and Management, v.89, p.189-95, 1996.

SOUZA, A.Q.L. et al. Atividade antimicrobiana de fungos endofíticos isolados de plantas tóxicas da Amazônia: Palicourea longiflora (aubl.) rich e Strychnos cogens bentham. Acta Amazônica, v.34, n.2, p.185-95, 2004.

SOUZA, M.L. Utilização de microorganismos na agricultura: uso de agentes microbianos na agricultura brasileira. Revista Biotecnologia Ciência \& Desenvolvimento, n.21, p.28-31, 2001. Disponível em: <http://www.bioteconologia.com.br>. Acesso em: 17 abr. 2006.

STIERLE, A.; STROBEL, G.A.; STIERLE, D. Taxol and taxane production by Taxomyces andreanae. Science, 
v.260, p.214-6, 1993.

STONE, J.K.; POLISHOOK, J.D.; WHITE JUNIOR, J.F. Endophytic fungi. In: MUELLER, J.M.; BILLS, G.F.; FOSTER, M.S. Biodiversity of fungi: inventory and monitoring methods. San Diego: Elsevier Academic Press, 2004. p.241-70.

STROBEL, G. et al. Stegolerium kukenani gen, et sp. nov. an endophytic taxol producing fungus from the

Roraima Stegolepsis guianensis and Kukenan tepuis of Venezuela. Mycotaxon, v.78, p.353-61, 2001.

SUTTON, B.C. The coelomycetes: fungi imperfecti with pycnidia acervuli and stromata. Kew, Surrey, England: Commonwealth Mycological Institute, 1980. 696 .

VEIGA JÚNIOR, V.F.; PINTO, A.C.; MACIEL, M.A.M. Plantas medicinais: cura segura? Química Nova, v.28, p.519-28, 2005. Disponível em: <http://www.scielo.br>. Acesso em: 27 jul. 2006. 